\title{
Electronic topological transition and noncollinear magnetism in compressed hcp Co
}

\author{
Y. O. Kvashnin, W. Sun, I. Di Marco, and O. Eriksson \\ Department of Physics and Astronomy, Division of Materials Theory, Uppsala University, Box 516, SE-75120 Uppsala, Sweden
}

(Received 27 August 2015; published 29 October 2015)

\begin{abstract}
Recent experiments showed that Co undergoes a phase transition from the ferromagnetic hcp phase to the nonmagnetic fcc one around $100 \mathrm{GPa}$. Since the transition is of first order, a certain region of coexistence of the two phases is present. By means of ab initio calculations, we found that the hcp phase itself undergoes a series of electronic topological transitions (ETTs), which affects both elastic and magnetic properties of the material. Most importantly, we propose that the sequence of ETTs lead to the stabilization of a noncollinear spin arrangement in highly compressed hcp Co. Details of this noncollinear magnetic state and the interatomic exchange parameters that are connected to it are presented here.
\end{abstract}

DOI: 10.1103/PhysRevB.92.134422

PACS number(s): 71.20.Be, 75.30.Et, 81.40.Vw

\section{INTRODUCTION}

By the development of the diamond anvil cells, it became possible to carry out high pressure experiments up to a few hundred GPa [1]. Such compressions roughly correspond to the state of the matter close to the Earth's core, so that the processes occurring under the deep mantle can be reconstructed in present day laboratories [2,3]. Particularly, the investigation of magnetic systems is of interest, because it can give fundamental insights on the origins of the geomagnetism [4].

Cobalt is not a particularly abundant element in the Earth's core and is therefore not intensively studied under extreme conditions. However, it is an outstanding element from technological and scientific viewpoints. At ambient conditions, Co crystallizes in an hep lattice showing a ferromagnetic $(\mathrm{FM})$ order with a high Curie temperature $\left(T_{c}\right)$ of $1388 \mathrm{~K}$. Its Fermi surface (FS) is highly spin-polarized, which makes it a great spin-filter material. The hcp phase can be stable up to a very high pressure, but at around $100 \mathrm{GPa}$ an hcp to fcc transition takes place $[5,6]$. There is an on-going debate about the magnetic state of cobalt above this critical pressure. Iota et al. proposed that Co remains in the magnetic hcp phase at least up to $100 \mathrm{GPa}$. For higher pressures, hcp Co is gradually transformed into the nonmagnetic (NM) fcc phase, which identifies a region of $50 \mathrm{GPa}$ where the two phases coexist and Co gradually loses its magnetism [7]. On the other hand, Torchio et al. argued that the magnetism is already completely lost at $120 \mathrm{GPa}$ in the mixed phase [8]. Ishimatsu et al. found a similar transition pressure but suggested the existence of a super paramagnetic (PM) fcc phase above 135 GPa [6]. However, all aforementioned groups analyzed the K-edge $\mathrm{X}$-ray magnetic circular dichroism (XMCD) data. This type of experiment does not probe directly the spin moment $\left(M_{S}\right)$ but rather the weak moment of the $4 p$ states [9]. Thus, some small values of the total magnetization might appear as a negligible noise on the spectra.

Theoretically, the problem of magnetism of Co under pressure has been extensively investigated by means of the density functional theory (DFT) [10-15]. Nevertheless, the precise transition pressure is uncertain, which can be traced back to differences among the employed DFT implementations [16]. The most recent calculations indicate that the fcc phase favors a NM state before the transition, while the hcp phase remains magnetic up to more than $150 \mathrm{GPa}$ [17].
On the experimental side, the pressure-induced anomalies of the $c / a$ ratio [18] and Raman frequencies [19] were attributed to a strong magnetoelastic coupling. However, any unequivocal evidence for the coupling between magnons and phonons have not been presented yet. In this paper, we point out that the above-mentioned peculiarities result from an electronic topological transition (ETT), or the so-called Lifshitz $2 \frac{1}{2}$ transition [20]. We also argue that the change of the FS gives rise to a noncollinear magnetic ground state in hcp Co under pressure and that this may be an excellent way to experimentally detect the influence of the ETT.

The paper is organized as follows. We start by describing the computational methods used in this work in Sec. I. In Sec. II we discuss the changes in the band structure of hep Co under pressure. Section III contains the results for elastic and magnetic properties and their relation to the ETTs. Effective exchange interactions and resulting magnetic excitation spectra are discussed in Sec. IV. The results of the noncollinear magnetic calculations are presented in Sec. V. Finally, the conclusions are discussed in Sec. VI.

\section{METHODS}

In the present study we have used several DFT implementations. The structural optimization was performed using the Vienna $a b$ initio simulation package (VASP) [21,22]. A plane-wave energy cutoff of $600 \mathrm{eV}$ and dense k-point grid containing $45 \times 45 \times 27$ points were used. Next, the calculations for the FS and the effective exchange parameters $\left(J_{i j}\right)$ were performed by means of the full-potential linear muffin-tin orbital method (FP-LMTO) as implemented in the RSPT code [23]. The $J_{i j}$ 's were computed between the $3 d$ states, projected on the muffin-tin spheres (see Ref. [24] for details). Additional simulations of the spin spirals (SS) were performed via the PY-LMTO code [25]. The results were obtained with the GGA-PBE [26] exchange correlation functional, since it reproduces the experimental hcp-fcc transition pressure $[12,13]$. We did not consider the effects of strong correlations, since it would require us to know not only the value of the Hubbard $U$ parameter but also its pressure dependence. Moreover, in our study we have to deal with very high pressures, where the large band broadening should diminish the correlation effects. Spin-orbit coupling is neglected throughout the paper, however we discuss how it possibly affects the results. 


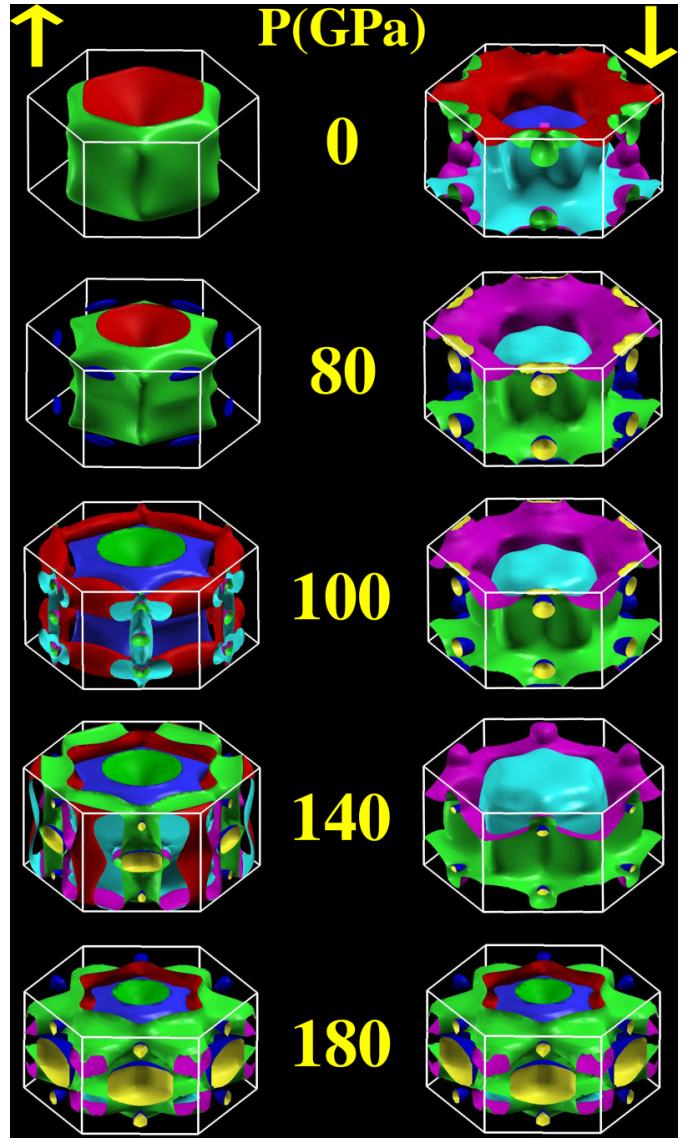

FIG. 1. (Color online) Fermi surface of the majority-spin ( $\uparrow$ ) and minority-spin $(\downarrow)$ electrons in hcp Co for different values of external pressure (in GPa). The results were obtained with the GGA-PBE functional. The plot was produced with the XCRYSDEN [27] software.

\section{BASIC ELECTRONIC STRUCTURE}

The calculated FS of the ferromagnetic hcp Co as a function of pressure is shown in Fig. 1. At ambient conditions, four minority bands and two majority-spin ones cross the Fermi level $\left(E_{F}\right)$. The shape of the FS is barely changed up until roughly $80 \mathrm{GPa}$. Above $80 \mathrm{GPa}$, a sequence of ETTs happen at different pressures in the spin-up channel: At around 80, 100 , and $140 \mathrm{GPa}$, one more band is counted to contribute to the $E_{F}$. At $180 \mathrm{GPa}$ the NM state is reached, which is characterized by all $d$ bands crossing $E_{F}$.

Close to $80 \mathrm{GPa}$ a particularly important ETT takes place. Many small sheets emerge in the spin-up channel, and the part of the FS close to the $\Gamma$ point of the spin-down channel is also significantly modified. To have more detailed information about the regions of the Brillouin zone (BZ) where the changes occur, we have calculated the $k$-resolved spectral function in hcp Co for different pressures. The results are shown in Fig. 2.

An inspection of Fig. 2 confirms that most of the ETTs occur for spin-up bands. Above $80 \mathrm{GPa}$ there are several transitions particularly along the M-L direction in the BZ, which is parallel to the $z$ axis. These transitions create a larger overlap between majority and minority states. As will be shown later, this peculiarity of the band structure leads to the stabilization of a particular family of spin spirals.

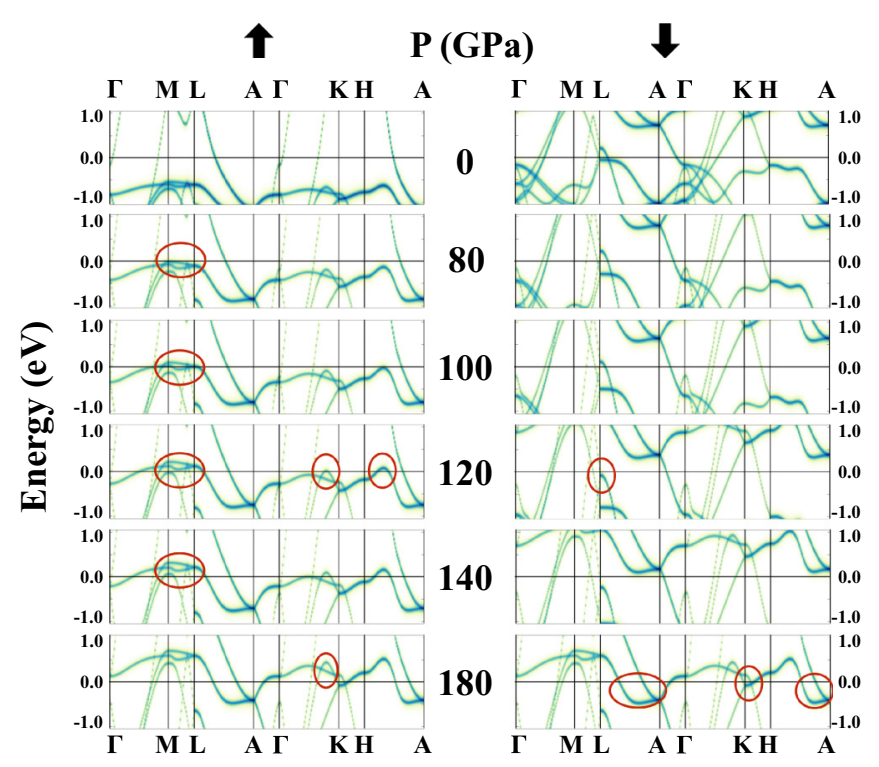

FIG. 2. (Color online) Computed $\vec{k}$-resolved spectral functions of the ferromagnetic hcp Co at different pressures. Spin-up bands are shown on the left side of the plot, while spin-down states are on the right. The results were obtained with the RSPT code. Red circles underline the bands, which cross the FS at different compressions. The $E_{F}$ is set to zero.

\section{ELASTIC AND MAGNETIC PROPERTIES}

Although there is no direct experimental evidence of this ETT, the changes in the topology of the FS can explain the changes that have been observed in several other quantities at around $80 \mathrm{GPa}$. First of all, at around this pressure, the $c / a$ ratio alters its pressure derivative, as was unveiled by Antonangeli et al. [18], who confirmed it both experimentally and theoretically. Second, vibrational properties such as, e.g., sound velocities show anomalous behavior at around $75 \mathrm{GPa}$ [28]. This anomaly is accompanied by a change of the slope of the $E_{2 g}$ phonon modes, which was first measured by Goncharov et al. (Ref. [19]) and later on reproduced in the DFT calculations [13]. Moreover, previous DFT studies [14,17,18] suggested that at the same compression ratio, i.e., $V / V_{0} \approx 0.8$, the pressure-driven reduction of the $M_{S}$ becomes faster.

In Fig. 3 we show the pressure dependence of the magnetic moment $\left(M_{S}\right), c / a$ ratio, and elastic constants $\left(C_{i i}\right.$ 's) along with the corresponding values of the density of states (DOS) at the $E_{F}$. The trends of the $M_{S}$ and $c / a$ are very similar to the one obtained by Antonangeli et al. (Ref. [18]). First of all, one can see that below $70 \mathrm{GPa} \operatorname{DOS}\left(E_{F}\right)$ is weakly dependent on the applied pressure. At $80 \mathrm{GPa}$, a small hump is visible, which is a manifestation of the ETT, as discussed previously. After the ETT, the DOS $\left(E_{F}\right)$ quickly surges upon compression. From Fig. 3(b) it is also seen that at low pressure, hcp Co has a relatively weak pressure dependence of the $M_{S}$. This is a consequence of the majority-spin band being completely occupied (see Ref. [29]). However, as we have shown above (Fig. 1), at around $80 \mathrm{GPa}$ the ETT pushes up the majority-spin band towards $E_{F}$. This causes a transition from a strong ferromagnet to a weak one, which makes the $M_{S}$ more vulnerable to the lattice compression. The resulting decrease 


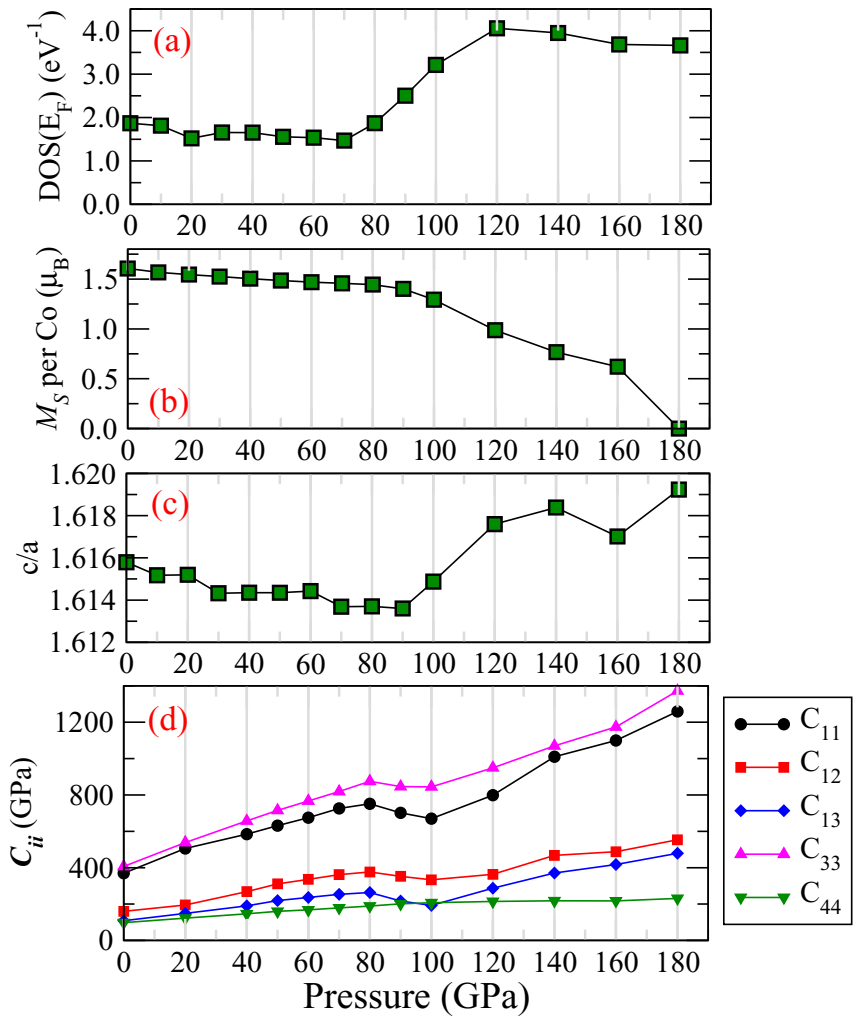

FIG. 3. (Color online) Pressure dependence of several ground state properties: DOS at the $E_{F}(\mathrm{a})$, magnetic moment $\left(M_{S}\right)$ per Co atom (b), $c / a$ ratio (c), and elastic constants (d).

of the $M_{S}$ is the primary reason for the destabilization of the FM hcp phase at high pressures. This is similar to the bcc-hcp transition in $\mathrm{Fe}$, which can be explained by mapping of the DFT solution on a simple Stoner model [30].

Signatures of the ETT can also be found in the elastic properties. For instance, our results indicate that an anomaly in the $c / a$ ratio occurs simultaneously with the jump of DOS at $E_{F}$, as seen in Fig. 3(c). The calculated elastic constants are reported in Fig. 3(d). As illustrated in Ref. [31], the elastic constants are directly related to the derivative of the DOS at the $E_{F}$. In the case of hcp Co we observe that all elastic constants except $C_{44}$ change their trends around $80 \mathrm{GPa}$, in agreement with prior studies [15]. From Fig. 3 it becomes apparent that this is a result of the remarkable change of the DOS $\left(E_{F}\right)$, associated with the ETT.

In the literature, the anomalies in the magnetic, elastic, and vibrational properties of Co were associated with a magnetoelastic coupling $[13,14,18,19,28]$. In our paper we provide strong evidence that all these changes are consequences of the ETTs. The present scenario is analogous to the one proposed for PM hcp Fe [32] and hcp Os [33]. In Ref. [32] it was reported that the only way to observe the ETT was to augment the DFT solution with the dynamical mean field theory [34]. The latter one significantly improves the description of the PM state as compared to the bare DFT, due to its proper account of the local moment fluctuations, which lead to strong damping of the quasiparticles. Our study, however, concerns the ordered phase where the many-body effects are less pronounced [35].
Note that ETTs do not necessary lead to the anomalies in the $c / a$ ratio. For example, the authors of Ref. [33] experimentally observed the $c / a$ anomaly in hcp Os but did not reproduce it in their DFT calculations. They suggested that the anomaly manifests itself only at finite temperatures due to the anisotropy in thermal expansion coefficients, whereas DFT describes the ground state at $T=0$. We suspect that one of the reasons for this is that in hep Os the value of the DOS $\left(E_{F}\right)$ is smoothly dependent on the pressure even across the ETT [36]. In our case these changes are more conspicuous [see Fig. 3(a)]. Moreover, it was recently shown that hcp Co has a rather strong electronphonon coupling [37]. In our opinion, a combination of these two factors in hcp Co allows the ETT to strongly influence the lattice-related properties of the material.

\section{EXCHANGE PARAMETERS AND ADIABATIC MAGNON SPECTRA}

As we have shown above, in the range of 80-180 GPa the hcp phase of Co remains magnetic and its $M_{S}$ strongly depends on the volume. However, the stability of the FM state at such conditions has never been examined. To shed light on this, we have calculated the effective $J_{i j}$ parameters by means of the magnetic force theorem [38].

The exchange parameters within the first few neighboring shells, calculated at various pressures, are shown in Fig. 4 (upper panel). The zero-pressure values are in good agreement with prior DFT studies [39,40]. Between 0 and $80 \mathrm{GPa}$ the nearest-neighbor couplings $\left(J_{1}\right)$ remain almost unchanged. This difference is instead most pronounced for the $J_{6}$ inter-
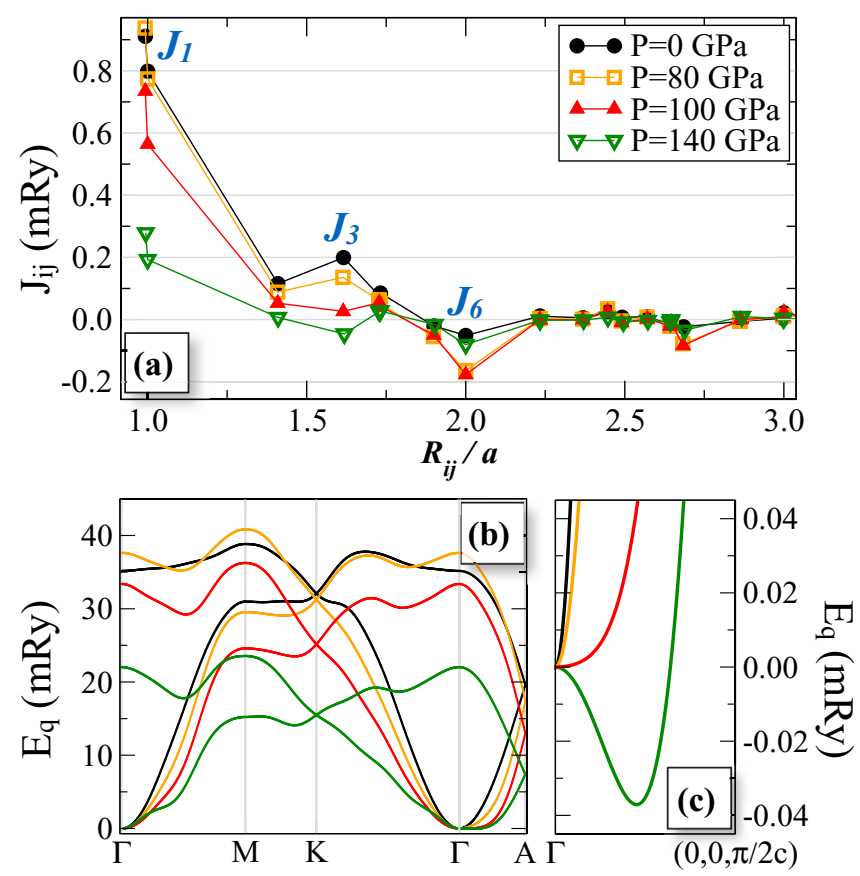

FIG. 4. (Color online) Panel (a): Intersite exchange parameters in hep Co as a function of distance for several values of pressure. The $J_{i j}$ 's are extracted for the Heisenberg model with unitary spins (see, e.g., Ref. [24].) Panel (b): Calculated adiabatic magnon spectra, obtained using the $J_{i j}$ 's shown in (a). Panel (c): Enlarged low-energy part of the magnon spectra along the $\Gamma$-A direction, where the softening appears. 
action: It is antiferromagnetic at ambient conditions and gets strongly enhanced at $80 \mathrm{GPa}$. This is the primary reason for the emergence of magnon softening along the $\Gamma$-A direction, as illustrated in the adiabatic magnon spectra reported in the panels (b) and (c) of Fig. 4. Further compression leads to vast modifications of the exchange parameters. Ferromagnetic $J_{1}$ and $J_{3}$ couplings become suppressed, and the latter one eventually changes sign. At $140 \mathrm{GPa}$ the $M_{S}$ is about $0.7 \mu_{B}$ per atom, i.e., the system is still magnetic, but its FM state is clearly unstable. The global minimum is formed at around $\vec{q}=(0,0,0.23 \pi / c)$, which is along the $\Gamma$-A (or $z$ ) direction.

\section{NONCOLLINEAR MAGNETIC GROUND STATES}

The instabilities in the magnon spectra, obtained above a certain pressure, indicate that the long-wavelength excitations destroy the FM ground state. What is peculiar is that they have only been found along the $\Gamma$-A path in the BZ, which motivated us to simulate the single- $\vec{q}$ family of the magnetic states. We have performed a series of noncollinear DFT calculations for the SS configurations by means of the generalized Bloch theorem [41]. Any SS state is characterized by two parameters: propagation vector $(\vec{q})$ and the cone angle between $\vec{q}$ and the magnetization $(\Theta)$. In Fig. 5 we show the relative total energies of the SS states with respect to the FM one.

An inspection of Fig. 5 suggests that at low compressions (e.g., $30 \mathrm{GPa}$ ) any SS state has a higher energy than that of the FM solution. This is consistent with the stable magnon spectrum in this regime. However, this behavior is changed as the pressure is raised up to $90 \mathrm{GPa}$. In a certain range of $\vec{q}$ vectors the SS states become energetically more favorable. The family of states with $\Theta=90$ degrees are the most stable ones. Further compression shifts the total energy minimum towards higher $\vec{q}$ values, and at $130 \mathrm{GPa}$ it reaches $-0.05 \mathrm{mRy}$ as compared with the FM state. We have calculated SS's with the $\vec{q}$ along other directions, but no solutions with lower energy

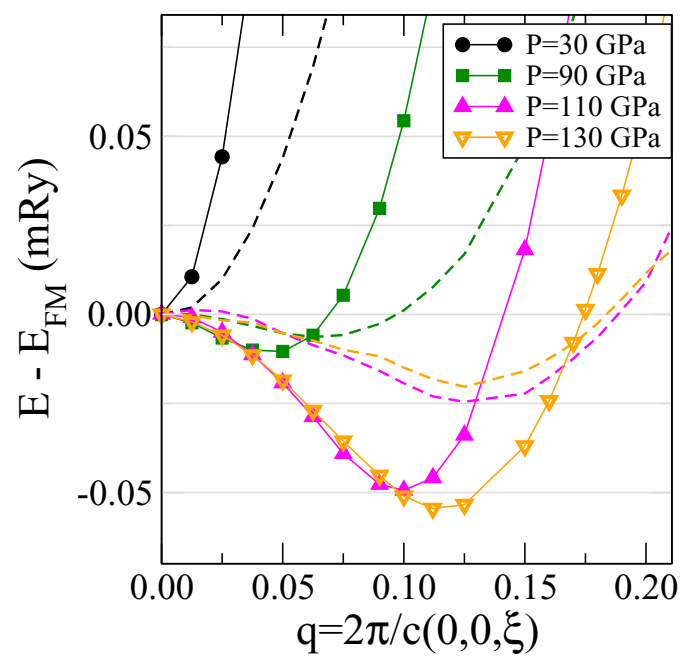

FIG. 5. (Color online) Calculated total energies (relative to that of the FM state) of the spin spiral states propagating along the $\Gamma-\mathrm{A}$ direction. At each pressure the calculations for two different cone angles $(\Theta)$ were performed. Dashed lines correspond to $\Theta=30$ degrees, solid ones to $\Theta=90$ degrees. No peculiarities were found in other directions, and thus they are not shown. were found. As we have mentioned above, the ETTs at $80 \mathrm{GPa}$ occur predominantly for the bands between $\mathrm{M}$ and $\mathrm{L}$ points in the BZ (see Fig. 2). This direction is parallel to $\Gamma-\mathrm{A}$, which most likely makes this direction particularly favorable for the propagation of the SS's.

Thus we suggest that the noncollinear states should be present in compressed hcp Co. In fact, it was previously proposed that SS configurations can be stabilized in all ferromagnetic transition metals upon compression [42]. However, this situation is very seldom observed experimentally, due to the presence of the structural transitions and/or the collapse of the $M_{S}$ at lower pressures. According to Ref. [42], the occurrence of the SS states is governed solely by the FS topology. In particular, it is absolutely necessary to have both spin-up and spin-down bands crossing $E_{F}$. We believe that hcp Co under pressure is a prototype system, where the ideas, proposed in Ref. [42], are realized. As we have shown above, at around $80 \mathrm{GPa}$ the system becomes a weak ferromagnet, which leads to the stabilization of the SS state.

Our results indicate that the behavior of cobalt under pressure is in many ways reminiscent of that of hcp Fe. In its PM state the $c / a$ anomaly was shown to originate from the pressure-driven ETT [32]. Moreover, the emergence of SS states was also suggested [43,44]. Extensive investigation of hcp Fe was partially motivated by the discovery of superconductivity below $1.5 \mathrm{~K}$ [45]. A close similarity of the two systems implies that an experimental investigation of the lowtemperature properties of hcp Co would be of high interest.

In relation to the K-edge XMCD experiments $[6,8]$ it is worth mentioning that a 90-degree SS state should not show any magnetic dichroism. On the other hand, an application of the external magnetic field is necessary in these experiments. Large fields, in principle, could lead to a different cone angle of the spiral. Our results suggest that, even if $\Theta$ reaches 30 degrees, these SS states will be more favored than the FM one (Fig. 5). Such a state will already be characterized by a nonzero XMCD signal, since the total $M_{S}$ is finite.

At this point it is important to discuss the relativistic effects, so far neglected in our study. Most importantly, spin orbit coupling gives rise to the magnetocrystalline anisotropy energy (MAE). At ambient conditions the MAE of hcp Co is $4.77 \mu \mathrm{Ry}$ per atom [46], which is ten times smaller than the maximal difference between SS and FM states. Moreover, stable SS's were found above $80 \mathrm{GPa}$, where the orbital moment is strongly suppressed, since it attenuates faster than the $M_{S}[9,47]$. The MAE is supposed to follow the orbital moment, since their relation is known [48]. Thus, we are confident that our conclusions remain valid in the case of finite spin-orbit coupling.

\section{CONCLUSIONS}

In summary, we have analyzed the Fermi surface and the band structure of hcp Co at different pressures by means of first principles calculations. We showed that the system undergoes a sequence of ETTs upon compression. The most important transition occurs at $80 \mathrm{GPa}$, which gives rise to a large increase in the DOS at the $E_{F}$. This transition is suggested to be the reason for the anomalies in various elastic properties, previously observed experimentally. Our analysis of the exchange couplings and the adiabatic magnon spectra 
indicates that upon compression the FM state gets destabilized. An instability in the magnon spectrum was shown to develop for particular $\vec{q}$ vectors, parallel to the $z$ direction. The self-consistent DFT calculations confirm that above $80 \mathrm{GPa}$, the spin spiral states propagating along the same direction become stable. We show that the noncollinear magnetism in compressed hcp Co is a result of the pressure-induced ETT.

\section{ACKNOWLEDGMENTS}

The authors acknowledge the computational resources provided by the Swedish National Infrastructure for Computing (SNIC) and Uppsala Multidisciplinary Center for Advanced Computational Science (UPPMAX). Support from Knut and Alice Wallenberg Foundation (KAW), Swedish Research Council (VR), and eSSENCE are acknowledged.
[1] R. Boehler, Rev. Geophys. 38, 221 (2000).

[2] L. Dubrovinsky, N. Dubrovinskaia, O. Narygina, I. Kantor, A. Kuznetzov, V. B. Prakapenka, L. Vitos, B. Johansson, A. S. Mikhaylushkin, S. I. Simak, and I. A. Abrikosov, Science 316, 1880 (2007).

[3] A. Dewaele, M. Torrent, P. Loubeyre, and M. Mezouar, Phys. Rev. B 78, 104102 (2008).

[4] B. M. Clement and L. Stixrude, Earth Planet. Sci. Lett. 130, 75 (1995).

[5] C. S. Yoo, H. Cynn, P. Söderlind, and V. Iota, Phys. Rev. Lett. 84, 4132 (2000).

[6] N. Ishimatsu, N. Kawamura, H. Maruyama, M. Mizumaki, T. Matsuoka, H. Yumoto, H. Ohashi, and M. Suzuki, Phys. Rev. B 83, 180409 (2011).

[7] V. Iota, J.-H. P. Klepeis, C.-S. Yoo, J. Lang, D. Haskel, and G. Srajer, Appl. Phys. Lett. 90, 042505 (2007).

[8] R. Torchio, A. Monza, F. Baudelet, S. Pascarelli, O. Mathon, E. Pugh, D. Antonangeli, and J. P. Itié, Phys. Rev. B 84, 060403 (2011)

[9] R. Torchio, Y. O. Kvashnin, S. Pascarelli, O. Mathon, C. Marini, L. Genovese, P. Bruno, G. Garbarino, A. Dewaele, F. Occelli, and P. Loubeyre, Phys. Rev. Lett. 107, 237202 (2011).

[10] C.-S. Yoo, P. Söderlind, and H. Cynn, J. Phys.: Condens. Matter 10, L311 (1998).

[11] G. Steinle-Neumann, L. Stixrude, and R. E. Cohen, Phys. Rev. B 60, 791 (1999).

[12] J. E. Saal, S. Shang, Y. Wang, and Z.-K. Liu, J. Phys.: Condens. Matter 22, 096006 (2010).

[13] P. Modak, A. K. Verma, R. S. Rao, B. K. Godwal, and R. Jeanloz, Phys. Rev. B 74, 012103 (2006).

[14] G. Steinle-Neumann, Phys. Rev. B 77, 104109 (2008).

[15] F.-G. Kuang, X.-Y. Kuang, S.-Y. Kang, and A.-J. Mao, Phys. B (Amsterdam, Neth.) 441, 72 (2014).

[16] The results of Ref. [14] indicate that the hcp phase loses its magnetism during the hcp-fcc transition, which is in contradiction with all other DFT-based studies.

[17] B. Kong, T.-X. Zeng, H.-B. Xu, D. liang Chen, Z.-W. Zhou, and Z.-J. Fu, Comput. Mater. Sci. 104, 130 (2015).

[18] D. Antonangeli, L. R. Benedetti, D. L. Farber, G. SteinleNeumann, A. Auzende, J. Badro, M. Hanfland, and M. Krisch, Appl. Phys. Lett. 92, 111911 (2008).

[19] A. F. Goncharov, J. Crowhurst, and J. M. Zaug, Phys. Rev. Lett. 92, 115502 (2004).

[20] I. M. Lifshitz, Zh. Eksp. Teor. Fiz. 38, 1569 (1960) [Sov. Phys. JETP 11, 1130 (1960)]

[21] G. Kresse, M. Marsman, and J. Furthmüller, "Vasp: Vienna abinitio simulation package", http://cms.mpi.univie.ac.at/VASP/ (2007).

[22] G. Kresse and D. Joubert, Phys. Rev. B 59, 1758 (1999).
[23] J. M. Wills, O. Eriksson, M. Alouani, and D. L. Price, in Electronic Structure and Physical Properies of Solids, Lecture Notes in Physics, Vol. 535, edited by H. Dreysse (Springer, Berlin Heidelberg, 2000), pp. 148-167.

[24] Y. O. Kvashnin, O. Grånäs, I. Di Marco, M. I. Katsnelson, A. I. Lichtenstein, and O. Eriksson, Phys. Rev. B 91, 125133 (2015).

[25] V. Antonov, B. Harmon, and A. Yaresko, Electronic Structure and Magneto-Optical Properties of Solids (Kluwer Academic, Dordrecht, 2004).

[26] J. P. Perdew, K. Burke, and M. Ernzerhof, Phys. Rev. Lett. 77, 3865 (1996).

[27] A. Kokalj, Comput. Mater. Sci. 28, 155 (2003).

[28] D. Antonangeli, M. Krisch, G. Fiquet, J. Badro, D. L. Farber, A. Bossak, and S. Merkel, Phys. Rev. B 72, 134303 (2005).

[29] P. Mohn, Magnetism in the Solid State: An Introduction, Springer Series in Solid-State Sciences (Springer, Berlin Heidelberg, 2003).

[30] O. Andersen, J. Madsen, U. Poulsen, O. Jepsen, and J. Kollár, Physica B+C 86, 249 (1977).

[31] M. I. Katsnelson, I. I. Naumov, and A. V. Trefilov, Phase Trans. 49, 143 (1994).

[32] K. Glazyrin, L. V. Pourovskii, L. Dubrovinsky, O. Narygina, C. McCammon, B. Hewener, V. Schünemann, J. Wolny, K. Muffler, A. I. Chumakov, W. Crichton, M. Hanfland, V. B. Prakapenka, F. Tasnádi, M. Ekholm, M. Aichhorn, V. Vildosola, A. V. Ruban, M. I. Katsnelson, and I. A. Abrikosov, Phys. Rev. Lett. 110, 117206 (2013).

[33] L. Dubrovinsky, N. Dubrovinskaia, E. Bykova, M. Bykov, V. Prakapenka, C. Prescher, K. Glazyrin, H. P. Liermann, M. Hanfland, M. Ekholm, Q. Feng, L. V. Pourovskii, M. I. Katsnelson, J. M. Wills, and I. A. Abrikosov, Nature (London) 525, 226 (2015).

[34] G. Kotliar, S. Y. Savrasov, K. Haule, V. S. Oudovenko, O. Parcollet, and C. A. Marianetti, Rev. Mod. Phys. 78, 865 (2006).

[35] A. Grechnev, I. Di Marco, M. I. Katsnelson, A. I. Lichtenstein, J. Wills, and O. Eriksson, Phys. Rev. B 76, 035107 (2007).

[36] D. Koudela, M. Richter, A. Möbius, K. Koepernik, and H. Eschrig, Phys. Rev. B 74, 214103 (2006).

[37] M. J. Verstraete, J. Phys.: Condens. Matter 25, 136001 (2013).

[38] A. Liechtenstein, M. Katsnelson, V. Antropov, and V. Gubanov, J. Magn. Magn. Mater. 67, 65 (1987).

[39] M. van Schilfgaarde and V. P. Antropov, J. Appl. Phys. 85, 4827 (1999).

[40] I. Turek, J. Kudrnovský, V. Drchal, P. Bruno, and S. Blügel, Phys. Status Solidi B 236, 318 (2003).

[41] L. M. Sandratskii, J. Phys.: Condens. Matter 3, 8565 (1991). 
[42] R. Lizárraga, L. Nordström, L. Bergqvist, A. Bergman, E. Sjöstedt, P. Mohn, and O. Eriksson, Phys. Rev. Lett. 93, 107205 (2004).

[43] V. Thakor, J. B. Staunton, J. Poulter, S. Ostanin, B. Ginatempo, and E. Bruno, Phys. Rev. B 67, 180405 (2003).

[44] R. Lizárraga, L. Nordström, O. Eriksson, and J. Wills, Phys. Rev. B 78, 064410 (2008).
[45] K. Shimizu, T. Kimura, S. Furomoto, K. Takeda, K. Kontani, Y. Onuki, and K. Amaya, Nature (London) 412, 316 (2001).

[46] M. Stearns, in 3d, 4d and 5d Elements, Alloys and Compounds, Landolt-Börnstein - Group III Condensed Matter, Vol. 19a, edited by H. Wijn (Springer, Berlin Heidelberg, 1986), pp. 41-47.

[47] R. Torchio (unpublished).

[48] P. Bruno, Phys. Rev. B 39, 865 (1989). 